\title{
EL COACHING: UNA FORMA PARA FORTALECER EL PROFESIONALISMO DEL DOCENTE EN EL AULA
}

\section{Coaching: a way to strengthen the professionalism of teachers in the classroom}

http://dx.doi.org/10.22235/pe.v9i2.1294

\author{
RAÚL JESÚS ZEGARRA HUAMÁN* \\ MÍRIAM ENCARNACIÓN VELÁZQUEZ TEJEDA*
}

Recibido: 12-04-2016

Revisado: 30-05-2016

Aceptado: 14-06-2016

Resumen: El profesionalismo del docente integra conocimientos y habilidades para el desempeño eficiente de la actividad pedagógica en el desarrollo del proceso de enseñanza-aprendizaje. Es una investigación educacional que se fundamenta en una perspectiva integradora, que conjuga dialécticamente los métodos cuantitativos y cualitativos para el estudio y análisis del fenómeno educativo en todas sus dimensiones. Como parte del diagnóstico, se aplicaron métodos e instrumentos que permitieron constatar la realidad del actuar de los docentes de las zonas rurales del Perú en el desempeño del proceso de enseñanza-aprendizaje. La sistematización de los referentes científicos, teóricos y metodológicos permitió triangular los datos y como conclusión esencial, diseñar un programa de aprendizaje basado en el coaching para fortalecer el profesionalismo del docente desde la autorreflexión sobre su desempeño en la práctica pedagógica del aula.

\footnotetext{
* Institución Educativa Ramiro Prialé Prialé. Distrito de Suitucancha, La Oroya, Junín, Perú.

${ }^{* *}$ Universidad San Ignacio de Loyola, Lima, Perú.
} 
Palabras clave: profesionalismo, desempeño, práctica pedagógica, coaching

\begin{abstract}
Professionalism of teachers integrates the knowledge and skills for the satisfactory performance of the educational activity to be achieved with permanent improvement to maintain efficiency in the development of teaching-learning process. Methodologically it is based on the qualitative approach applied educational type. Were applied methods for diagnostic, techniques and tools that enabled us to confirm the reality that demonstrates the act of teachers in rural areas of Peru and its performance in the teaching - learning process. The systematization of the related scientific, theoretical and methodological helped design the program based learning Coaching $A B C$, to strengthen the professionalism of teachers in the performance of pedagogical practice in the classroom.
\end{abstract}

Keywords: professionalism, performance, teaching practice, coaching

\title{
INTRODUCCIÓN
}

La educación es un proceso social que tiene por encargo la formación integral del ser humano para la vida, de manera que se autotransforme permanentemente y contribuya a la transformación del contexto. Ello exige de los sistemas educativos enfrentar eficazmente los procesos formativos e incidir en los problemas sociales como la multicultura, la tecnología, las necesidades de convivencia, la multiétnica, la universalización de los esfuerzos en contra al cambio climático, la conservación del planeta y el ser humano. Se hace imprescindible desarrollar la educación para la formación crítica solidaria, creativa, tecnológica, y las aulas abiertas o globales (Ferrer, 1998).

En cualquier sistema educativo el docente es el centro de la escuela. Por su efecto multiplicador, es el profesional encargado de formar a las nuevas generaciones acorde al desarrollo de las ciencias, la tecnología, los valores humanos y humanistas que mueven la actitud, según las motivaciones y las 
exigencias sociales. Debe mostrar un profesionalismo óptimo, que le permita integrar los conocimientos y la maestría en el desempeño de su función social y se concrete en la eficiencia educativa como un proceso permanente. Al respecto Ospina, Toro y Aristizábal (2008), expresan:

Es necesario que el docente asuma una visión compleja de la enseñanza y el aprendizaje [...], que le permita tanto a él como al estudiante aplicar los conocimientos en diversos contextos y plantear soluciones a los problemas que plantea la cotidianidad y el ejercicio profesional (p. 113).

En esa línea, el Ministerio de Educación del Perú, en el Marco del Buen Desempeño Docente (2015), establece en el cuarto dominio, competencia 8 y desempeño 36: "Reflexiona en comunidades de profesionales sobre su práctica pedagógica e institucional y el aprendizaje de todos sus estudiantes", y en el 37 agrega: "Participa en las experiencias significativas del desarrollo profesional en concordancia con sus necesidades, las de los estudiantes y la de la escuela" ( $p$. 49).

Se pretende un mejoramiento continuo del profesionalismo del docente desde la autorreflexión y participación activa a través de las experiencias de aprendizaje colegiadas, de donde emerge la apropiación de los conocimientos científico-pedagógicos que le permiten alcanzar los saltos cualitativos en el desempeño del proceso enseñanza-aprendizaje para que se reflejen en la calidad del aprendizaje de los estudiantes de cada nivel educacional (Nemiña, García y Montero, 2009).

Sin embargo, en la práctica pedagógica de los docentes de las zonas rurales se evidencian serias insuficiencias, en el nivel de profesionalismo teórico y metodológico, para desempeñarse en la dirección del proceso de enseñanzaaprendizaje de manera de responder a las necesidades y demandas cognitivas, afectivas y volitivas de los estudiantes y así contribuir a su formación integral para la vida. 
Se encuentra una contradicción externa entre las exigencias del nivel de profesionalismo que deben alcanzar los docentes de las zonas rurales en su práctica pedagógica y las insuficiencias que, en este sentido, se constatan en el desempeño del proceso de enseñanza-aprendizaje. Este problema orienta el estudio de la presente investigación, cuyo objetivo es proponer el Programa de Aprendizaje basado en el Coaching - ABC, para fortalecer el profesionalismo del docente de la zona rural en el desempeño de la práctica pedagógica en el aula.

El proceso investigativo responde a un llamado del Ministerio de Educación de Perú dirigido a realizar propuestas para mejorar la práctica pedagógica desde el programa de maestría cursado en la Universidad San Ignacio de Loyola en 2015.

\section{FUNDAMENTO TEÓRICO}

El fortalecimiento del profesionalismo del docente desde el aula se concibe, en la propuesta presentada, a partir del análisis y la autorreflexión en interacción con sus colegas como vía para la apropiación consciente de los conocimientos. Esto se realiza mediante un proceso de orientación, debate, asesoría y demostración por parte de un especialista con el que el docente interactúa recíprocamente y así transita a niveles superiores de desempeño. Ello se basa en las posiciones conceptuales de la categoría reflexión dialógica e interacción social, abordadas por Copello y Sanmartí (2000), que citan a Vigotsky y Wertsch al definir la interacción social como la estrategia desarrollada para facilitar la reelaboración del conocimiento pedagógico en la práctica del docente.

Es un proceso orientado a que el docente se apropie de las herramientas necesarias para el autoanálisis voluntario y la autocrítica de su desempeño profesional, con vista a incorporarlo en su quehacer pedagógico como parte de un proceso de perfeccionamiento progresivo contextualizado.

Lev Vigotsky, en la Teoría Sociocultural, ofrece el marco de referencia para asumir la interacción social como un proceso que posibilita el intercambio, 
socialización, enriquecimiento teórico de ideas y experiencias de los individuos. Esa concepción, llevada al campo de la superación del docente en su aula asistida por un especialista, trae como consecuencia un enriquecimiento de experiencias, de conocimientos y un fortalecimiento de las competencias profesionales para un desempeño idóneo en la práctica pedagógica.

La profesionalidad del docente se evidencia a través del dominio de la ciencia que enseña (conocimientos, habilidades profesionales y valores morales), dominio de los métodos de enseñanza-aprendizaje y de formación de valores, y los resultados prácticos acumulados en la labor educativa (Chacón, 1999).

Dentro de las habilidades profesionales está la competencia comunicativa, que es esencial para su desempeño. El desarrollo del lenguaje, la expresividad, la calidad de las ideas, la asertividad y la empatía, tanto individual como en el acto pedagógico, son funciones que demuestran la maestría pedagógica alcanzada. El docente debe tener los recursos didácticos para saber cómo orientar a los estudiantes a expresar sus ideas, opiniones, puntos de vista, reconocer sus fortalezas, sus carencias, sin inhibiciones, y proporcionarles las herramientas básicas para cambiar su realidad.

En general, se requiere de un cambio cualitativo superior en los procesos formativos y la superación permanente del docente. En tal sentido, el trabajo asistido por un especialista competente es viable por varias razones. En esta modalidad el protagonista es el docente en el espacio concreto del aula, donde reflexiona acerca de los logros, las deficiencias, los retos a enfrentar y, con la ayuda del especialista, reflexiona, evalúa su desempeño, busca las alternativas de solución y las aplica según sus necesidades, las de su grupo y las del contexto.

\section{Antecedentes}

Como antecedente se tuvo en cuenta a Píriz (2013) cuando analiza la perspectiva del docente en ejercicio y como educador social, y asevera que debe producir conocimientos en la enseñanza y, de manera general, en su profesionalidad. 
Johns (2008) emplea la metodología cualitativa y el coaching entre docentes de manera muy diversa, al apreciar las diferencias culturales, los niveles de alfabetización heterogéneos que poseen, el enriquecimiento de experiencia y la disposición al cambio.

La investigación realizada por Kebaetse (2010) da a conocer la necesidad de aplicar una técnica de trabajo asociado, y al respecto desarrolla cuatro métodos de colaboración: modelado, exploración, reflexión y coaching. Lo anterior se concreta en una investigación centrada en el alumno, mediante la observación y la entrevista.

Ledesma (2010), en su estudio, aplica el coaching en cómo orientar a los estudiantes a descubrirse a sí mismos mediante el diálogo, y nota una influencia en su desarrollo lingüístico y en su inteligencia interpersonal.

Balzan (2008) hace alusión al coaching o mentoring y emplea el término tutoring en su relación con la supervisión educativa y el acompañamiento pedagógico como alternativa al rol del docente y del supervisor.

Cárdenas (2011), en su estudio de aplicación del coaching, obtuvo influencias positivas en el desarrollo del desempeño de las funciones de los profesores de la provincia de Huancayo, Perú.

Como se aprecia, existen estudios y experiencias exitosas que demuestran la transformación cualitativa que se experimenta durante la aplicación del coaching como alternativa para la superación del docente desde su puesto de trabajo, en el que identifica fortalezas, debilidades y estrategias de solución para poner en práctica en su desempeño profesional.

\section{MARCO CONCEPTUAL}

\section{El profesionalismo docente}

La profesión docente es reconocida por la humanidad debido al valor y trascendencia que tiene en la educación integral de los niños, adolescentes y jòvenes para su inserción en la sociedad (ONU, 1948). La definición elemental de 
profesión como empleo, ejercicio de un oficio por el cual se percibe una retribución de tipo económico (Real Academia Española, n.d.) es insuficiente para definir el alcance y proyección de esta tarea, que es una obra de vocación y amor infinito por el estudiante.

El docente necesita desarrollar al máximo sus competencias, habilidades y destrezas (Díaz, 2005) por la misma complejidad de su labor, que influye directamente en la formación de la personalidad del estudiante. Debe irradiar autoridad, respeto, sabiduría, asertividad, afecto y humanidad para lograr la concentración y formación de los estudiantes.

La docencia es una profesión que va más allá del mero cumplimiento del deber de la clase diaria. No es un acto de misericordia, tampoco una dádiva o favor humano: el rol del docente tiene que basarse en las ciencias de la educación y centrarse en las potencialidades y necesidades del educando para contribuir a su transformación cualitativa para vida (Ascorra y Crespo, 2004).

El profesionalismo del docente en el aula

La sala de clase es el escenario principal donde se evidencia el nivel de profesionalismo del docente a través de su desempeño. Es allí donde pone en práctica su maestría pedagógica: el dominio del contenido, el método de enseñanza, la orientación idónea, la atención diferenciada, la problematización del contenido, la comunicación asertiva, la autoridad, el prestigio y la ejemplaridad.

El accionar del docente responde a la ética que la profesión lleva en sí misma. El acto pedagógico se da en el aula a través de la clase como célula básica del proceso de enseñanza-aprendizaje, donde se dan las contradicciones dialécticas que conducen al desarrollo de los conocimientos teóricos y prácticos, así como las transformaciones del estudiante. Es un espacio donde confluyen los esfuerzos, la sabiduría por enseñar y la motivación por aprender, en un proceso interactivo dialéctico y complejo. El docente debe poner en práctica todo su potencial en su labor para generar aprendizajes duraderos, reflexivos, eficientes y eficaces. La realización de su vocación se da en el ejercicio de su desempeño con profesionalismo (Hargreaves, 1996). 
Profesionalismo y profesionalidad

Entre ambas deficiones conceptuales existe una diferencia sutil, como lo hace saber Hargreaves (1996), al enfatizar que el profesionalismo está concebido como el mayor o menor grado de preparación que tiene un profesional para desenvolverse según las actitudes, funciones y normas de la actividad profesional, mientras que la profesionalidad se entiende como el proceso que busca elevar el prestigio académico integral y el mejoramiento social de los que desempeñan la profesión. Pone énfasis en considerar a la persona y la necesidad de la sociedad, la aplicación y el empeño en la construcción de una imagen positiva de sí y de la misma profesión que desempeña.

En consecuencia, no se basa solo en el deber de ser exigido desde afuera; tiene que ver con su convicción, grado de desarrollo y ejercicio profesional realizado con vocación, agrado y afectividad. Va más allá de su deber ser y lo trasciende hasta lograr una característica única y ejemplarizante, como lo requieren las actuales circunstancias de la educacion pública peruana (Oliveira, 2013).

\section{La profesionalidad docente}

La profesionalidad docente integra los conocimientos y las habilidades necesarias para el desempeño de su función social con la obtención de resultados prácticos satisfactorios, así como los valores humanos y humanistas que mueven la actitud y conducta que se asume en la labor y ante la sociedad (Chacón, 1999). A ello hay que agregarle la reconquista de su autoestima, la valoración de las experiencias significativas, la autorreflexión sobre su desempeño y la búsqueda de soluciones a los problemas de su labor pedagógica.

\section{DEFINICIÓN Y METODOLOGÍA}

La metodología empleada en el proceso investigativo se fundamenta en la investigación educacional que desde una perspectiva integradora conjuga dialécticamente los métodos cuantitativos y cualitativos para el estudio y análisis 
del fenómeno educativo en todas sus dimensiones. Fraile y Vizcarra (2009) aseguran que en este enfoque, el docente parte del conocimiento de su experiencia en el aula, accede a la teoría, la sistematiza dialécticamente y la contrasta con la realidad, para lograr una comprensión objetiva del problema, sus causas y consecuencias. De esa manera, se apropia de una concepción científica que le permite, desde una visión holística, conceptualizar la realidad pedagógica y proponer alternativas de solución al problema objeto de estudio.

En esta investigación se utilizaron diferentes métodos, técnicas e instrumentos, como el método histórico-lógico, análisis y síntesis, y el hermenéutico, que permitieron un conocimiento holístico del objeto estudiado. Como técnicas se emplearon la entrevista semiestructurada a los docentes, el análisis documental, la observación a clase, la modelación para el diseño de la propuesta, el criterio de experto para la validación del programa. Como procedimientos se validaron las guías de la entrevista semiestructurada, con los documentos necesarios de la gestión y control de la práctica educativa, y se tramitaron las autorizaciones correspondientes; la guía de observación a clase, donde se filmaron las acciones que realizaron los docentes en la sesión de aprendizaje y un listado de cotejo para la revisión del registro de ocurrencias; la consulta de especialista para validar el Programa de Aprendizaje basado en el Coaching - $\mathrm{ABC}$ como producto científico de orden práctico aportado por la investigación. Mediante la triangulación se realizó el procesamiento de la información (Bisquerra, 2004) para establecer las regularidades e identificar las barreras que inciden en el desarrollo del profesionalismo del docente para desempeñar con eficiencia el proceso de enseñanza-aprendizaje en el aula.

\section{CONTEXTO}

\section{La institución educativa}

La Institución Educativa Ramiro Prialé Prialé se ubica en la localidad de Suitucancha, provincia de Yauli, en Junín, en el Perú. Se localiza en la vertiente occidental de la cordillera de los Andes centrales a 4255 metros sobre el nivel del 
mar, en la región Jalca. El distrito se extiende hasta los 5730 de altitud en la región Janca o Cordillera (Pulgar, 1999).

\section{Los docentes}

Los docentes que realizan la labor pedagógica en esta escuela rural muestran una gran complejidad: la mayoría provienen de otras localidades como Huancayo, Jauja, Junín, Tarma y Lima. En las entrevistas, refieren que la asesoría y la capacitación metodológica que deben realizar los funcionarios estatales no están diseñadas para ellos, debido a diferentes realidades objetivas y subjetivas de la misma zona rural.

Enfatizan que los especialistas metodológicos no siempre están dispuestos a asistir a esas zonas a desarrollar el trabajo, por el difícil acceso a esos enclaves rurales. Las condiciones del contexto de actuación le exigen al docente de la escuela rural aplicar el conocimiento pedagógico especializado ante algunos temas que requieren de una adecuada conducción, como la interculturalidad, la inclusión, normas lingüísticas, grupos étnicos, y allí demostrar eficacia profesional. Pero si solo tienen las herramientas básicas, muy poco pueden hacer ante la realidad. Al respecto enfatiza Encinas (1932) cuando se refiere a que la falta de competencia de los maestros tiene como origen que los centros formadores no cumplen con esa demanda.

En la escuela rural existen docentes con un nivel de profesionalismo probado en la actividad pedagógica. Orientarlos por un programa como el que se presenta, basado en la metodología del coaching, puede ser una alternativa para que, desde su puesto de trabajo, reflexionen acerca de su práctica e identifiquen los problemas y las soluciones que deben aplicar en el proceso de enseñanzaaprendizaje de la escuela rural.

\section{ORIGEN DE LA PROPUESTA Y SENTIDO DEL ESTUDIO}


Con el estudio y análisis del problema investigado se ha realizado una valoración de la metodología del Coaching, que permite adaptarla para proponer el Programa de Aprendizaje basado en el Coaching - ABC como forma de fortalecer el profesionalismo del docente en su desempeño.

El programa propuesto está concebido para implementarse en diferentes etapas, de forma que respete la libertad, el compromiso, los estilos de aprendizaje y la enseñanza de los docentes de la escuela rural. Se ha diseñado según los resultados del diagnóstico de entrada, con el fin de contribuir al profesionalismo del docente desde la autorreflexión de su labor en el aula. Se pone énfasis en el aspecto procedimental y los resultados esperados que establecen los documentos normativos institucionales, así como en el compromiso de los docentes y estudiantes.

Los docentes que se inicien en el programa recibirán una asesoría permanente desde el puesto de trabajo por parte de un especialista que los asesorará para que, mediante la autorreflexión, les permita adquirir la competencia profesional que deberán revertir en su desempeño profesional al conducir la enseñanza e identificar las necesidades de los estudiantes, los ritmos, los estilos de aprendizaje y los niveles de desarrollo integral que estos demuestren.

Las acciones de entrenamiento se corresponderán con el enfoque pedagógico asumido por el docente para atender la inclusión social y a los estudiantes con habilidades diferentes a su cargo. Estos requerimientos son exigidos por norma, pero el docente no tiene las competencias para desplegar su accionar pedagógico ante cada caso, se ve impotente ante el problema, la familia y la sociedad, que le otorgan una responsabilidad para la cual no ha recibido adiestramiento.

El programa busca que el docente se apropie de las herramientas pedagógicas e investigativas para identificar y resolver problemas de la práctica pedagógica mediante la aplicación del método científico. Los docentes de las zonas rurales necesitan entrenarse en la investigación de su quehacer profesional, que demanda equidad, justicia social, convivencia, tolerancia, respeto y calidad educativa en su desempeño. Ese profesionalismo se agiganta cuando, en esas 
El coaching: una forma para fortalecer el profesionalismo del docente en el aula

comunidades montañosas, el docente no solo es el educador que enseña, sino es el médico, el sociólogo que ofrece charlas, el que orienta cómo prevenir la salud y el que irradia cultura en la zona.

El programa está diseñado para contribuir al profesionalismo eficiente de los docentes desde la autorreflexión en las tres dimensiones de la profesión: la orientación educativa, la dirección del proceso de enseñanza-aprendizaje y la investigación como una herramienta para transformar su práctica educacional. 


\section{RESPUESTA A LA PROBLEMÁTICA: QUÉ ES Y POR QUÉ EL COACHING}

\section{Algunas experiencias de la aplicación del coaching con fines educativos}

Los estudios dan cuenta de cómo el coaching se ha aplicado en la formación del profesorado con resultados impactantes en la práctica docente. Jiménez (2012, p. 243) cita a Saphier y West quienes refieren que el coaching es una estrategia para el éxito de la escuela y el desarrollo de los estudiantes. Su concepción está orientada a la formación continua del profesorado; se basa en la escuela colaborativa y está centrada en el aprendizaje autorreflexivo y colegiado del docente y su efecto transformador en los estudiantes.

El uso de esta tecnología no es reciente. El trabajo de Joyce y Showers (1984) da fe de la aplicación del coaching en función de la preparación del profesorado. Hargreaves y Dawe (1990) afirman que es una estrategia novedosa y creativa, y su aplicación en la práctica se extiende hasta la actualidad.

Los estudios de Wise y Avendaño (2013) demuestran que el "coaching permite llevar a una persona valiosa de donde él o ella está a donde él o ella pueden llegar" (p.11). El proceso interactivo, socializador y sistemático le permite al entrenador asistir a su receptor para desencadenar sus capacidades y conseguir lo mejor de sí mismo. Bisquerra (2008) complementa este circuito, al enfatizar la acción del coaching como medio de relación entre estos dos actores esenciales.

Sánchez y Bonorat (2014) destacan el sustento psicológico, filosófico y pedagógico de esta estrategia, al establecer una relación de similitud con la mayéutica socrática en cuanto al proceso de indagación e interrogación que se realiza con el docente para que reflexione sobre su problemática individual en el aula y su experiencia, y para que proponga soluciones con iniciativas loables, lo que va exteriorizando su potencial profesional pedagógico.

El aporte de la psicología humanista y la teoría pedagógica permite considerar la necesidad de relacionarse con libertad, confianza y empatía, para que los sujetos participantes trabajen sin grandes barreras por alcanzar objetivos 
de alto impacto en el proceso de descubrimiento y ejercitación comunicativa permanentes (Bou, 2013; Ruiz, 2013).

La diversidad geográfica del Perú exige la incorporación de nuevas estrategias y metodologías para lograr el profesionalismo docente de las zonas rurales y provocar impactos desarrolladores en el desempeño en el aula. Es preciso aplicar los aportes de las investigaciones pedagógicas para contribuir a la transformación de ese tipo de escuela para que influya decisivamente en la formación de los estudiantes y ayudar al cambio cultural de los pobladores de esas zonas (Cisterna Cabrera, 2005; Barrientos y Navío, 2015; Gajardo y De Andraca, 1992; López, 2012; Vaillant, 2007).

El coaching que se propone en el programa diseñado tiene por objetivo que el docente de la zona rural, asesorado por un especialista, desarrolle sus competencias pedagógicas en los contextos especiales de actuación profesional, cuya necesidad le permita reflexionar, encontrar soluciones y un eficiente profesionalismo (Goldsmith y Lyons, 2010; Hudson, 1999; Whitmore, 2012; Bou, 2013).

\section{EL PROCESO DE DIAGNÓSTICO DE CAMPO Y SUS RESULTADOS}

Se realizó el diagnóstico de campo para constatar la realidad del problema objeto de investigación. Este consistió en la aplicación de diferentes métodos, técnicas e instrumentos como la entrevista a docentes, observación a clases, el registro de las incidencias apreciadas en una sesión de clases y análisis de documentos normativos de la Institución Educativa Ramiro Prialé Prialé, de Suitucancha, seleccionada como muestra.

El análisis cuantitativo y cualitativo realizado a partir de la aplicación y procesamiento de la información obtenida revelan un deficiente nivel de profesionalismo del docente en su desempeño y muestran deficiencias de orden teórico, didáctico e investigativo en el tratamiento a los distintos momentos de la clase. Los docentes consideran que la realidad de los estudiantes obedece a diversos factores de orden familiar, del contexto y de su propia preparación, pues 
no se sienten competentes para asumir la docencia en varios grados a la vez. Los estudiantes reflejan serias deficiencias en el rendimiento del aprendizaje, muy por debajo de las exigencias de los objetivos del grado que cursan.

\section{PROPUESTA DEL PROGRAMA DE APRENDIZAJE BASADO EN EL COACHING}

La sistematización teórica y los resultados del diagnóstico hicieron posible proponer un Programa de Aprendizaje basado en el Coaching - ABC, para fortalecer el profesionalismo del docente de la zona rural. En este modelo el docente reflexiona acerca de su práctica y accede a aprendizajes significativos que revierte en la conducción del proceso de enseñanza-aprendizaje, primero asistido por un especialista y luego, en la medida que alcanza las competencias requeridas, de manera independiente, para demostrar el profesionalismo alcanzado (Rogers, 1981).

Se parte de la concepción de la teoría humanista acerca del aprendizaje al explicar que es un proceso que se da como fruto de la observación, el diálogo y la reflexión en espacios de socialización, compartiendo experiencias y con la participación entre colegas. Es un aprendizaje profesional que parte de lo social a lo personal, mediante un proceso recíproco y cíclico hacia el interior del docente que asimila, procesa, comprende, valora y luego pasa a la exteriorización. Estas premisas sirven de base a la concepción del programa, que considera al docente como protagonista de su propia transformación mediante un proceso dirigido a lograr aprendizajes eficientes y que deja de lado la visión de receptores de la enseñanza (Casanova, 1989).

\section{Procesos}

De acuerdo a estos procesos generales, se proponen los siguientes momentos en la aplicación del coaching:

Exploración de la situación actual, inicial 
El coaching: una forma para fortalecer el profesionalismo del docente en el aula

- Proceso de razonamiento

Descripción de la situación deseada

- Establecimiento de objetivos

- Mapa de opciones y recursos

Diseño de acciones efectivas

- Información científica valida, suficiente y actual

Realización de acciones

Entrenamiento - ejercitación, simulación y práctica

- Para dominio inicial

- Para dominio experto

- Para logro destacado

Verificación de resultados, reflexiones finales y cierre

- Comunicación de logros

- Publicación de logros ${ }^{1}$

\footnotetext{
${ }^{1}$ Adaptado de Wolk, 2007, p. 115.
} 


\section{El modelo analógico del programa}

Figura 1: El modelo del programa Fuente: Zegarra, 2015. 
Las etapas se muestran a continuación en las siguientes tablas:

\section{Tabla 01}

\section{Etapa 0: Inducción}

Contiene la estructura básica de la etapa de inducción del Programa ABC y todo lo concerniente a la evolución adecuada del programa.

Fuente: Zegarra, 2015. 
Tabla 02

Adecuación y sesiones de la etapa de inducción

Contiene la diversificación de la etapa de inducción al Programa $A B C$ y las cuatro sesiones que se desarrollan en esta etapa.

Fuente: Zegarra, 2015. 
Figura 2: Adecuando la inducción al programa Fuente: Zegarra, 2015. 


\section{Tabla 03}

\section{Fase I: Programa de entrenamiento ocho semanas}

Contiene la estructura del programa, en su desarrollo durante ocho semanas, y los aspectos que se realizan en procesos iterativos para el logro de resultados.

Fuente: Zegarra, 2015.

\section{Ciclo del programa}

Figura 3: Ciclo de vida del programa

Fuente: Zegarra, 2015 
El coaching: una forma para fortalecer el profesionalismo del docente en el aula ¿Cómo elegir el tema de trabajo o la competencia docente a desarrollar?

Figura 4: Elección del tema a desarrollar en el entrenamiento del Programa ABC Fuente: Zegarra, 2015

Revista Páginas de Educación. Vol. 9, Núm. 2 (2016) ISSN: 1688-5287; e-ISSN: 1688-7468 
El coaching: una forma para fortalecer el profesionalismo del docente en el aula

\section{Forma de entrenamiento}

Figura 5: Procedimiento del entrenamiento en la sesión de reflexión docente

Fuente: Zegarra, 2015

Revista Páginas de Educación. Vol. 9, Núm. 2 (2016) ISSN: 1688-5287; e-ISSN: 1688-7468 
El coaching: una forma para fortalecer el profesionalismo del docente en el aula

\section{Niveles de logro del programa}

Figura 6: Plan de logros semanales del Programa de Aprendizaje basado en el Coaching - ABC.

Fuente: Zegarra, 2015

Revista Páginas de Educación. Vol. 9, Núm. 2 (2016) ISSN: 1688-5287; e-ISSN: 1688-7468 


\section{Publicación de resultados}

Figura 7: Publicación de resultados

Fuente: Zegarra, 2015

\section{Valoración de las potencialidades del programa por consulta a especialistas}

Para validar la efectividad del Programa de Aprendizaje basado en el Coaching $A B C$ se empleó el método de consulta a especialistas mediante una ficha que recogió los aspectos internos y externos del producto científico.

Este método tiene diferentes requerimientos para su aplicación, por ello se diseñaron dos fichas de valoración y se eligieron a los especialistas teniendo en cuenta los siguientes criterios: que poseyeran el grado de maestro o doctor en Ciencias de la Educación, que hubiesen trabajado en el área de formación docente y que hubieran sido docentes de educación superior pedagógica, con experiencia en la formación y superación de profesores y en la docencia de cursos de investigación científica. Los resultados finales de la valoración de los especialistas son los siguientes:

\section{Tabla 04}

\section{Valoración de los resultados}

Fuente: Zegarra, 2015

Los especialistas evaluaron el programa propuesto como muy bueno. En sus valoraciones aseveran que procede su aplicación en el contexto de la zona rural para la que se diseñó y dejan explícito que, dado los fundamentos teóricos y metodológicos del programa, podría analizarse su generalización a otras zonas y 
contextos, siempre que se tengan en cuenta las características psicopedagógicas, sociales y culturales del nivel o área donde se pretenda aplicar.

\section{CONCLUSIONES Y RECOMENDACIONES}

\section{Conclusiones}

El especialista Píriz (2013) afirma:

El docente (...) asume la enseñanza como la relación pedagógica en la que interactúa con los "fragmentos" disciplinares (...) con los estudiantes, estos entre sí y también con esos "fragmentos", reconoce que se trata de una relación que encierra una complejidad (p. 195).

En concordancia con ello, desde el punto de vista pedagógico, el programa diseñado le permite al docente reflexionar acerca de su realidad de aula y dirigir el proceso de enseñanza-aprendizaje a través de una didáctica desarrolladora que orienta el aprendizaje desde los niveles de desarrollo actual a los potenciales, y proporciona cambios en las formas de pensar, actuar y sentir de los estudiantes.

Johns (2008), desde su trabajo referido al coaching, anticipa: "El éxito educativo de los estudiantes depende de la preparación, las habilidades y la pedagogía del docente de aula"1 (p. 197). En concordancia con ese postulado, el aporte esencial de esta investigación consiste en el diseño del Programa de Aprendizaje basado en el Coaching - ABC, que contribuye a fortalecer el profesionalismo del docente desde una práctica pedagógica reflexiva que lo conduce a alcanzar las competencias profesionales necesarias para lograr la formación integral de los estudiantes.

Balzan (2008) afirma que "las funciones del coaching son necesarias para el ámbito educativo [...] el aprendizaje mutuo entre docentes y los supervisores". El Programa de Aprendizaje basado en el Coaching - ABC parte de la autorreflexión

\footnotetext{
${ }^{1}$ Traducido del inglés por los autores.
} 
y la autonomía del docente, en un marco de confianza y asertividad entre los participantes, que orienta a la objetividad en el trabajo.

El diagnóstico de campo demostró que el desarrollo del profesionalismo del docente en el aula tiene serios inconvenientes debido a la percepción subjetiva y personalista de su práctica profesional en el aula, por la falta de empatía y relaciones interpersonales asertivas con los supervisores y directivos.

Wise y Avendaño (2013) en el plano internacional y Cárdenas (2011) en el plano nacional reconocen el potencial de la aplicación de la metodología del coaching para la educación. Desde esta perspectiva se orientó la investigación, de cuyo proceso surgió como aporte el Programa de Aprendizaje basado en el Coaching - ABC para fortalecer el profesionalismo del docente de la zona rural desde la autorreflexión de su desempeño en la práctica pedagógica en el aula.

\section{Recomendaciones}

- Analizar las potencialidades del programa para su posible aplicación en los colegios de las zonas rurales, como una alternativa para contribuir al desempeño reflexivo del docente desde su aula, de modo que le permita alcanzar la eficiencia profesional en la práctica pedagógica.

- Validar el impacto del programa basado en la metodología del coaching, para constatar su pertinencia y efectividad en su puesta en práctica en el nivel de profesionalismo del docente y el desempeño en el proceso de enseñanza-aprendizaje, así como en la formación integral de los estudiantes.

\section{REFERENCIAS}

Ascorra, P. y Crespo, N. (2004). La incidencia del rol docente en el desarrollo del conocimiento metacomprensivo. Psicoperspectivas, III, 23-32. Recuperado de

http://www.psicoperspectivas.cl/index.php/psicoperspectivas/article/viewFile/ $10 / 10$ 
El coaching: una forma para fortalecer el profesionalismo del docente en el aula

Balzan, Y. (2008). Acompañamiento pedagógico del supervisor y desempeño docente en III etapa de Educación Básica. Maracaibo: Universidad Rafael Urdaneta.

Barrientos Delgado, C. y Navío Gámez, A. (2015). Formador de la educación superior técnico profesional: Percepciones sobre su trabajo docente. Estudios Pedagógicos, XLI (1), 45-61. Recuperado de http://www.scielo.cl/pdf/estped/v41n1/art03.pdf

Bisquerra Alcina, R. (2008). Coaching: Un reto para orientadores. Revista española de orientación y pedagogía, 19 (2), 163-170. Recuperado de http://www2.uned.es/reop/pdfs/2008/19-2\%20-

\%20Rafael\%20Bisquerra\%20Alzina.pdf

Bou, J. F. (2013). Coaching educativo. Bogotá: Lid.

Cárdenas Valverde, J. C. (2011). Coaching y desempeño docente en la provincia de Huancayo [Tesis]. Recuperado de http://es.calameo.com/read/0014179013219b4ae1f1e

Casanova, E. M. (1989). El proceso educativo según Carl R. Rogers: la igualdad y formación de la persona. Revista Interuniversitaria de Formación del Profesorado, 6, 599-603. Recuperado de https://dialnet.unirioja.es/servlet/articulo?codigo=117692

Cisterna Cabrera, F. (2005). Categorización y triangulación como procesos de validación del conocimiento en investigación cualitativa. Theoría, 14, 61-71. Recuperado de http://www.ubiobio.cl/theoria/v/v14/a6.pdf

Copello Levy, M. I. y Sanmartí Puig, N. (2000). Fundamentos de un modelo de formación permanente del profesorado de ciencias centrado en la reflexión dialógica sobre las concepciones y las prácticas. RACO: Revistes Catalanes amb Accés Obert. Recuperado de http://www.raco.cat/index.php/ensenanza/article/viewFile/21741/21574

Chacón Arteaga, N. (1999). Formación de valores morales. La Habana: Academia.

Díaz, M. (2005). Modalidades de enseñanza centradas en el desarrollo de competencias. Orientaciones para promover el cambio metodológico en el 
espacio europeo de educación superior. Universidad de Oviedo.

Recuperado de

http://www.uvic.es/sites/default/files/Ensenanza_para_competencias.PDF

Encinas, J. A. (1932). Un ensayo de escuela nueva en el Perú. Lima: Minerva.

Ferrer, F. (1998). Educación y sociedad: Una nueva visión para el siglo XXI. Revista Española De Educación Comparada, 4, 11-35.

Fraile, A. y Vizcarra, M. T. (2009). La investigación naturalista e interpretativa desde la actividad física y el deporte. Revista psicodidáctica, 14 (1), 119132. Recuperado de http://www.redalyc.org/articulo.oa?id=17512723008

Gajardo, M., \& De Andraca, A. M. (1992). Docentes y docencia, Las zonas rurales. (UNESCO/FLACSO, Ed.) Santiago, Chile: Orelac. Recuperado el 6 de 11 de 2015, de http://unesdoc.unesco.org/images/0009/000923/092388SB.pdf

Goldsmith, M. y Lyons, L. (2010). Coaching: La última palabra en desarrollo de liderazgo. New Jersey: Prentice Hall.

Hargreaves, A. (1996). Profesorado, cultura y post modernidad. Cambian los tiempos, cambia el profesorado. Madrid: Ediciones Morata.

Hargreaves, A. y Dawe, R. (1990). Paths of professional development: Contrived collegiality, collaborative culture, and the case of peer coaching. Teaching and Teacher Education, 6, (3), 227-241.

Hudson, F. (1999). The Handbook of Coaching, A comprensive Resource Guide for Managers, Executives, Consultants, and Human Resource Professionals. San Francisco: Jossey-Bass Publishers.

Jiménez, R. (2012). La investigación sobre coaching en formación del profesorado: Una revisión de estudios que impactan en la conciencia sobre la práctica docente. Profesorado, 16 (1), 238-252. Recuperado de http://www.redalyc.org/articulo.oa?id=56724377014

Johns, J. R. (2008). Abriendo caminos: Peer coaching of culturally relevant pedagogy for teachers of adolescent emergent bilinguals. Recuperado de http://search.proquest.com/docview/304661640

Joyce, B. y Showers, B. (2002). Student achievement through staff development. Alexandria: ASCD. 
El coaching: una forma para fortalecer el profesionalismo del docente en el aula

Kebaetse, M. B. (2010). Scaffolding techniques used by educational developers to support academics in the design of learner-centered courses. http://search.proquest.com/docview/366456606

Ledesma, M. (2010). Analisis del coaching para la inteligencia interpersonal de los estudiantes de educación media. [Tesis de maestría] Recuperado de http://dspace.ucuenca.edu.ec/handle/123456789/2817

López, M. (2012, 5 de julio). Ser Maestro en el Perú. El Comercio, p. 25.

Recuperado de http://elcomercio.pe/blog/huellasdigitales/2010/07/quesignifica-ser-maestro-en-e

MINEDU. (2015). Marco del Buen Desempeño Docente. Lima: MINEDU. Recuperado de http://www.perueduca.pe/documents/60563/ce664fb7-a1dd450d-a43d-bd8cd65b4736

Nemiña, R. E., García Ruso, H. y Montero Mesa, L. (2009). Desarrollo profesional y profesionalización docente. Perspectivas y problemas. Profesorado. 13 (2), 13. Recuperado de https://www.ugr.es/ recfpro/rev132COL3.pdf

Oliveira, H. (2013). La construcción de la profesionalidad docente. Revista Educación, 22 (42), 97-115. Recuperado el 19 de 01 de 2016, de http://revistas.pucp.edu.pe/index.php/educacion/article/view/5293

ONU. (1948). Declaración Universal de Derechos Humanos. Recuperado de http://www.un.org/es/documents/udhr/

Ospina, B. E., Toro, J. A. y Aristizábal, C. (2008). Rol del profesor en el proceso de enseñanza aprendizaje de la investigación en estudiantes de enfermería de la universidad de Antioquia, Colombia. Investigación y Educación en Enfermería, XXVI (1), 106-114. Recuperado de http://www.redalyc.org/articulo.oa?id=105215231010

Píriz, S. (2013). Producción de conocimiento y profesionalidad docente: La experiencia de los docentes - educadores sociales. [Tesis de Maestría]. Recuperado de http://posgrados.cse.edu.uy/sites/posgrados.cse.edu.uy/files/tesis_silvia_piri z.pdf

Pulgar, J. (1999). Geografía del Perú. Las ocho regiones naturales. Lima: PUCP. 
Real Academia Española. (n.d.). Profesión. Diccionario de la lengua española. Recuperado de http://dle.rae.es/?id=UHx86MW

Rogers, C. (1981). Psicoterapia centrada en el cliente. Barcelona: Paidós.

Ruiz, M. (2013). Coaching para mejorar el proceso de enseñanza-aprendizaje. Cuaderno de pedagogía universitaria, 10 (19), 14-17. Recuperado de https://issuu.com/pucmm.edu.do/docs/cuaderno_pedagog_a_universitaria_ $1 / 19$

Sánchez, M. y Bonorat, J. (2014). Coaching Educativo: Modelo para el desarrollo de competencias intra e interpersonales. Educación XXI, 17 (1), 221-242. Recuperado de http://revistas.uned.es/index.php/educacionXX1/article/view/10712

Vaillant, D. (2007). Mejorando la formación y el desarrollo profesional docente en Latinoamérica. Pensamiento Educativo, 41 (2). Recuperado de http://www.ub.edu/obipd/PDF\%20docs/Formaci\%C3\%B3\%20Permanent/Ed ucaci\%C3\%B3\%20Primaria/Publicacions/Mejorando\%20la\%20formaci\%C3 \%B3n\%20y\%20el\%20desarrollo\%20profesional\%20docente\%20en\%20Lati noam\%C3\%A9rica.\%20Vaillant,D.pdf

Whitmore, J. (2012). Coaching. El método para mejorar el rendimiento de las personas. Madrid: Paidós.

Wise, D. y Avendaño, K. (2013). Coaching para el liderazgo educativo. Guatemala: USAID/G. Recuperado de http://cnbguatemala.org/index.php?title=Coaching_para_el_liderazgo_educa tivo. 